\title{
Ferredoxin als Kofaktor der cyclischen Photophosphorylierung in einem zellfreien System aus der Blaualge Anacystis nidulans
}

\author{
HERMANN BOTHE \\ Ferredoxin-catalysed Cyclic Photophosphorylation in Lyophilised and Lysozyme \\ Treated Anacystis nidulans
}

Pflanzenphysiologisches Institut der Universität Göttingen, Abt. Biochemie der Pflanzen

(Z. Naturforschg. 24 b, 1574-1582 [1969] ; eingegangen am 9. August 1969)

\begin{abstract}
The properties of the ferredoxin-catalysed cyclic photophosphorylation in lyophilised and lysozyme treated Anacystis nidulans with rates up to $80 \mu$ moles ATP $/ \mathrm{h} / \mathrm{mg}$ chlorophyll are described. The concentration of ferredoxin needed for the saturation of the ferredoxin-catalysed cyclic photophosphorylation is about 6-8 times higher than for NADP-reduction. In contrast to the ferredoxin-catalysed cyclic photophosphorylation in spinach chloroplasts the reaction in Anacystis is relatively intensive to the "poising" of the system and is not affected by antimycin A. Whilst under aerobic conditions in broken chloroplasts ferredoxin catalyses a pseudocyclic photophosphorylation in which $\mathrm{H}_{2} \mathrm{O}_{2}$ is a component, in Anacystis on addition of ferredoxin ATP is only formed on the cyclic pathway even in air. In chloroplasts as well as in Anacystis disulfo-disalicylidenpropandiamin (DSPD) inhibits only the ferredoxin-catalysed cyclic photophosphorylations and the NADP-reductions, but not the ferredoxin stimulated ATP-formations under aerobic conditions. Evidence is presented once more that ferredoxin is the cofactor of cyclic photophosphorylation in vivo.
\end{abstract}

In isolierten Chloroplasten ${ }^{1}$ lassen sich nur dann cyclische Photophosphorylierungen nachweisen, wenn bestimmte Kofaktoren zu den Präparationen zugesetzt werden. Die Kofaktoren können natürlicher (wie Chinone oder FMN ${ }^{*}$ ) oder unphysiologischer (wie PMS oder DCPIP) Herkunft sein. Unter ihnen hat Ferredoxin besondere Bedeutung, da Ferredoxin wahrscheinlich der Kofaktor in vivo ist ${ }^{2}$. Die Ferredoxin-katalysierte cyclische Photophosphorylierung ist allerdings nur in isolierten Chloroplasten höherer Pflanzen nachgewiesen worden ${ }^{3}$, nicht aber in Präparationen aus Blaualgen, obwohl cyclische Photophosphorylierungen mit allen anderen Kofaktoren dort bekannt geworden sind ${ }^{4-8}$. In der Blaualge Anacystis nidulans läßt sich eine Ferredoxinkatalysierte cyclische Photophosphorylierung mit einer nicht unbeträchtlichen Rate in einer durch Lyophilisation und Lysozymbehandlung gewonne-

* Verwendete Abkürzungen: PMS = Methylphenazoniummethosulfat; $\mathrm{FMN}=$ Flavinmononucleotid; $\mathrm{ADP}$ und $\mathrm{ATP}=$ Adenosindi- und triphosphat $; \mathrm{DCMU}=$ Dichlorphenyldimethylharnstoff; NADP und $\mathrm{NADPH}_{2}=$ oxyd. und red. Nicotinamid-Adenin-Dinucleotidphosphat; Chl.= Chlorophyll; DSPD = Disulfo-disalicylidenpropandiamin ; DCPIP = Dichlorphenolindophenol.

1 D. I. Arnon, Science [Washington] 122, 3157 [1955].

2 D. I. Arnon, H. Y. Tsujimoto u. B. D. McSwain, Nature [London] 214, 562 [1967].

3 K. Tagawa, H. Y. Tsujimoto u. D. I. Arnon, Proc. nat. Acad. Sci. USA 49, 567 [1963]. nen Präparation nachweisen. Die Eigenschaften dieser Reaktion werden im folgenden beschrieben, und es sollen die Ferredoxin-katalysierten cyclischen Photophosphorylierungen in der Präparation aus Anacystis und in „broken chloroplasts“ miteinander verglichen werden.

\section{Methodik}

Die Anzucht der Blaualge Anacystis nidulans Richter (Stamm 1402-1 der Algensammlung des Pflanzenphysiologischen Institutes der Universität Göttingen) erfolgte in einer nach Kratz und MYers ${ }^{9}$ modifizierten Nährlösung (vgl. l. c. ${ }^{10}$ ) und in einer von LORENZEN ${ }^{11}$ beschriebenen Kulturanlage bei $35^{\circ} \mathrm{C}, 3000$ Lux und einer Begasung mit einem Kohlendioxyd-Luftgemisch $\left(1,5\right.$ Vol. $\left.\% \mathrm{CO}_{2}\right)$. Die Herstellung des zellfreien Systems aus Anacystis wurde im wesentlichen bereits beschrieben ${ }^{6}$. Algen mit einem $\mathrm{Na}$ ggewicht von 0,5 bis $0,6 \mathrm{~g}$ (aus der log. Wachstumsphase) wurden mit dest.

4 B. Petrack u. F. Lipmann, in: Light and Life, Hrsg. W. D. McElroy u. B. Glass, The Hopkins Press, Baltimore, 1961, S. 621.

5 W. C. Duane, M. C. Hohl u. D. W. Krogmann, Biochim. biophysica Acta [Amsterdam] 109, 108 [1965].

6 B. Gerhardt u. A. Trebst, Z. Naturforschg. 20 b, 879 [1965].

7 J. Biggins, Plant Physiol. 42, 1447 [1967].

8 S. S. Lee, A. M. Joung u. D. W. Krogmann, Biochim. biophysica Acta [Amsterdam] 180, 130 [1969].

9 W. A. Kratz u. J. Myers, Amer. J. Bot. 42, 282 [1955].

10 H. Bothe, Dissertation, Göttingen 1968.

11 H. Lorenzen, Flora [Jena] 147, 382 [1959]. 
Wasser gewaschen, nach der Zentrifugation bei $5000 \mathrm{~g}$ in 5-proz. Saccharose gelöst in $5 \mathrm{ml} 0,02 \mathrm{M} \mathrm{H}$ e p e s Puffer $\mathrm{pH} 7,0$ resuspendiert, bei $-60^{\circ} \mathrm{C}$ eingefroren und $2-3 \mathrm{Stdn}$. lyophilisiert. Unmittelbar darauf wurde das erhaltene Trockenpulver in $4 \cdot 10^{-2} \mathrm{M} \mathrm{MgCl}_{2}$ gelöst in $0,02 \mathrm{M} \mathrm{H}$ e p e s-Puffer $\mathrm{pH} \mathrm{7,0}$ aufgenommen und mit Lysozym (Calbiochem. Nr. 62783, 2 mg Lysozym/ $0,2 \mathrm{mg}$ Chlorophyll) versetzt. Die Wirkung von Lysozym auf die lyophilisierten Anacystis-Zellen wurde bereits früher beschrieben ${ }^{12}$. Die Lysozyminkubation erfolgte $90 \mathrm{Min}$. lang bei $25^{\circ} \mathrm{C}$ unter kontinuierlichem Schütteln. Danach wurden die aufgebrochenen Zellen entweder getestet (Präparation $\mathrm{N}_{\text {Lys }}$ ) oder bei $20000 \mathrm{~g}$ $5 \mathrm{Min}$. lang abzentrifugiert, aufgenommen in $0,02 \mathrm{M}$ $\mathrm{H}$ e p e s-Puffer pH 7,0, erneut bei $20000 \mathrm{~g} 5 \mathrm{Min}$. lang zentrifugiert und mit $0,02 \mathrm{M} \mathrm{H}$ e p e s - Puffer $\mathrm{pH}$ 7,0 (0,2 mg Chl./ml Puffer) suspendiert (Präparation $\mathrm{R}_{2}$ ).

Die Reaktionsansätze zur Bestimmung der photosynthetischen Aktivitäten der Präparationen aus Anacystis in konischen W a r burg-Gefäßen mit etwa $14 \mathrm{ml}$ Inhalt enthielten in einem Gesamtvolumen von $3 \mathrm{ml}$, wenn nicht anders angegeben: $1 \mathrm{ml}$ der Algenpräparation mit einem Chlorophyllgehalt von $0,2 \mathrm{mg}$, $100 \mu \mathrm{Mol} \mathrm{H}$ e p e s-Puffer pH 7,0, $40 \mu \mathrm{Mol} \mathrm{MgCl}_{2}$, $10 \mu \mathrm{Mol} \mathrm{ADP}, 10 \mu \mathrm{Mol} \mathrm{Na}_{2} \mathrm{HPO}_{4}$ radioaktiv markiert ( 100000 ipm ${ }^{32} \mathrm{P}$ ) und die in den Legenden der Tabn. bzw. Abbn. angegebenen Zusätze. Nach Äquilibrierung der Gefäße mit nachgereinigtem $\mathrm{N}_{2}$ bzw. Luft (5 Min.) wurden die Versuche bei $25^{\circ} \mathrm{C} 20 \mathrm{Min}$. lang bei 35000 Lux (Philips-Attralux-Lampen) im W a r bu r g-Apparat ausgeführt. In einigen Versuchen wurden zwischen der Lichtquelle und den W a r b u r g - Gefäßen im W a r b u r g-Apparat RGI-Filter, Schott und Gen. Mainz, befestigt, die Lichtintensität an den W a rburg-Gefäßen betrug dann $1,0 \cdot 10^{5} \mathrm{erg}$. $\mathrm{cm}^{-2} \cdot \mathrm{sec}^{-1}$.

Die Sauerstoffentwicklung wurde manometrisch verfolgt. Die gebildeten Mengen an $\mathrm{NADPH}_{2}$ wurden unmittelbar nach dem Ende des Versuches in einem Aliquot der bei $20000 \mathrm{~g} 5 \mathrm{Min}$. lang abzentrifugierten Proben durch die Messungen der Extinktionen bei $340 \mathrm{~m} \mu$ vor und nach Zugabe von $0,03 \mathrm{ml}$ einer PMS-Lösung (1 mg PMS $/ \mathrm{ml}$ ) zur Meßküvette und zur Vergleichsküvette ermittelt. Unmittelbar nach Entnahme des Aliquots für die $\mathrm{NADPH}_{2}$-Bestimmung wurden die Proben mit 0,4 ml 20-proz. Trichloressigsäure gestoppt. Das gebildete ATP wurde nach SugINo und MryoshI ${ }^{13}$ nach Fällung des anorganischen Phosphates mit Ammoniummolybdat über das in organisches Phosphat eingebaute ${ }^{32} \mathrm{P}$ an einem Endfensterzählrohr bestimmt.

Zur Bestimmung der $\mathrm{H}_{2} \mathrm{O}_{2}$-Bildung wurde ein Aliquot des Überstandes der abzentrifugierten Proben mit einer frisch hergestellten Titanylsulfat-Lösung versetzt und die optische Dichte des entstandenen Komplexes

12 B. Gerhardt, Ber. dtsch. bot. Ges. 78, 400 [1966].

13 Y. Sugino u. Y. Mryoshi, J. biol. Chemistry 239, 2360 [1964].

14 G. MacKinney, J. biol. Chemistry 140, 315 [1941]. bei $395 \mathrm{~m} \mu$ bestimmt. Aus einer Eichkurve mit $\mathrm{H}_{2} \mathrm{O}_{2}$ wurde der Extinktionskoeffizient mit $0,75 \mathrm{mMol}^{-1} \mathrm{~cm}^{-1}$ ermittelt.

Die Extraktionen und Mengenbestimmungen des Chlorophylls erfolgten in 80-proz. Aceton nach MAC KINNEY ${ }^{14}$ unter Verwendung eines Extinktionskoeffizienten von $82 \mathrm{mMol}^{-1} \mathrm{~cm}^{-1}$ bei $663 \mathrm{~m} \mu$. Die Versuche mit isolierten Chloroplasten, hergestellt nach ARNON ${ }^{1}$, wurden im Warburg-Apparat entsprechend wie die mit den Präparationen aus Anacystis durchgeführt. Ferredoxin aus Spinat wurde nach der von ARNON ${ }^{3}$ beschriebenen Methode gewonnen und hatte ein Verhältnis der optischen Dichten von $420 \mathrm{zu}$ $280 \mathrm{~m} \mu$ um 0,3. Die Herstellung von Ferredoxin aus Anacystis nidulans erfolgte im wesentlichen nach der von SMillie ${ }^{15}$ angegebenen Methode (vgl. l. c. ${ }^{10}$ ), das Verhältnis der optischen Dichten von $420 \mathrm{zu} 280 \mathrm{~m} \mu$ betrug 0,4-0,45. Zur Berechnung der Mengen an Ferredoxin wurde ein Extinktionskoeffizient von 10,32 $\mathrm{mMol}^{-1} \mathrm{~cm}^{-116}$ verwendet.

\section{Ergebnisse}

Neben Kofaktoren wie Menadion, PMS oder FMN ${ }^{6,17}$ katalysiert auch Ferredoxin eine cyclische Photophosphorylierung in Blaualgen. Das läßt sich bereits in lyophilisierter und Lysozym-behandelter Anacystis nidulans aber noch besser in nach der Lyophilisation und Lysozymbehandlung mit verdünntem Puffer extrahierten Algen zeigen. Durch die Extraktion werden das zugesetzte Lysozym und die wasserlöslichen Proteine der Algen, u. a. der größte Teil des Phycocyanins und des Ferredoxins von der Präparation entfernt. In der so erhaltenen Präparation wird bei Belichtung nur wenig ATP gebildet; erst nach Zusatz von Ferredoxin zum Versuchsansatz läßt sich eine ATP-Bildung bis zu $80 \mu \mathrm{Mol} / \mathrm{h} / \mathrm{mg}$ Chlorophyll messen (Tab. 1). Somit

\begin{tabular}{lc}
\hline & $\begin{array}{c}\text { gebildete } \\
\mu \text { Mol ATP }\end{array}$ \\
\hline ohne Kofaktor & 0,85 \\
+95 m $\mu$ Mol Ferredoxin, Dunkelkontrolle & 0,3 \\
+95 m $\mu$ Mol Ferredoxin & 4,1 \\
$+0,3 \mu$ Mol PMS & 6,4 \\
\hline
\end{tabular}

Tab. 1. Ferredoxin und PMS als Katalysatoren der cyclischen Photophosphorylierung in lyophilisierter, Lysozym-behandelter und extrahierter Anacystis. Reaktionsbedingungen: $3 \mathrm{ml}$ Reaktionsvolumen, Algen $\left(\mathrm{R}_{2}\right)$ mit $0,2 \mathrm{mg}$ Chl., Ferredoxin aus Anacystis, pH 7,0, $10^{-6} \mathrm{M}$ DCMU außer im Gefäß mit PMS, 20 Min. Licht bei 35000 Lux und $25^{\circ} \mathrm{C}$.

15 R. M. Smillie, Plant Physiol. 40, 1124 [1965].

16 F. R. Whatley, K. Tagawa u. D. I. Arnon, Proc. nat. Acad. Sci. USA 49, 266 [1963].

17 B. Gerhard u. R. Santo, Z. Naturforschg. 21 b, 673 [1966]. 
ist die Rate der Ferredoxin-katalysierten cyclischen Photophosphorylierung erheblich, sie ist unter den in Tab. 1 angegebenen Versuchsbedingungen nur um etwa $1 / 3$ geringer als die der PMS-katalysierten. Der Verlauf der Reaktion ist mindestens während 20 Min. linear.

Auch in isolierten Chloroplasten aus Spinat läßt sich eine Ferredoxin-katalysierte cyclische Photophosphorylierung erst nach Zusatz von Ferredoxin messen. Allerdings ist die Ferredoxin-katalysierte cyclische Photophosphorylierung in isolierten Chloroplasten nur nachzuweisen ${ }^{18,19,2}$ a) unter streng anaeroben Bedingungen, b) wenn Filter verwendet werden, die undurchlässig für kurzwelliges Licht sind, da möglicherweise Ferredoxin im Weißlicht inaktiviert wird ${ }^{20}$, c) wenn sich die an der cyclischen Photophosphorylierung beteiligten Kofaktoren in einem bestimmten Redoxzustand befinden. Dieser Redoxzustand kann experimentell dadurch erreicht werden, daß die Kofaktoren der cyclischen Photophosphorylierung durch den offenkettigen Elektronentransport reduziert werden. Allerdings darf der offenkettige Elektronentransport nur mit einer schwachen, genau bestimmten Rate ablaufen; wäre die Rate zu groß, würden die Kofaktoren der cyclischen Photophosphorylierung überreduziert werden, wäre sie zu gering, so wäre die Reduktion der Kofaktoren nicht ausreichend (,poising des Systems“"). Die benötigte Rate des offenkettigen Elektronentransportes kann durch Verwendung einer bestimmten Konzentration des Hemmstoffes DCMU eingestellt werden. In Abb. 1 ist nochmals dokumentiert, wie kritisch im Chloroplastensystem die DCMU-Konzentration ist; sie ändert sich stark mit den Versuchsbedingungen, etwa mit dem Chloroplastengehalt im Versuchsansatz. Im Vergleich zu den Verhältnissen in isolierten Spinatchloroplasten ist - unter gleichen Versuchsbedingungen - die Ferredoxin-katalysierte cyclische Photophosphorylierung in lyophilisierter Anacystis nur wenig DCMUabhängig (Abb. 1). Die Elektronenüberträger der Ferredoxin-katalysierten cyclischen Photophosphorylierung scheinen in der Präparation aus Anacystis bereits in einem geeigneteren Redoxzustand zu sein, so daß in dem System aus Anacystis ein ,poising“

18 D. I. ARnon, in: Biochemistry of Chloroplasts, Hrsg. T. W. Goodwin, Acad. Press,New York-London 1967, Vol. II, S. 461.

19 B. R. Grant u. F. R. Whatley, in: Biochemistry of Chloroplasts, Acad. Press, New York-London 1967, Vol. II, S. 505.

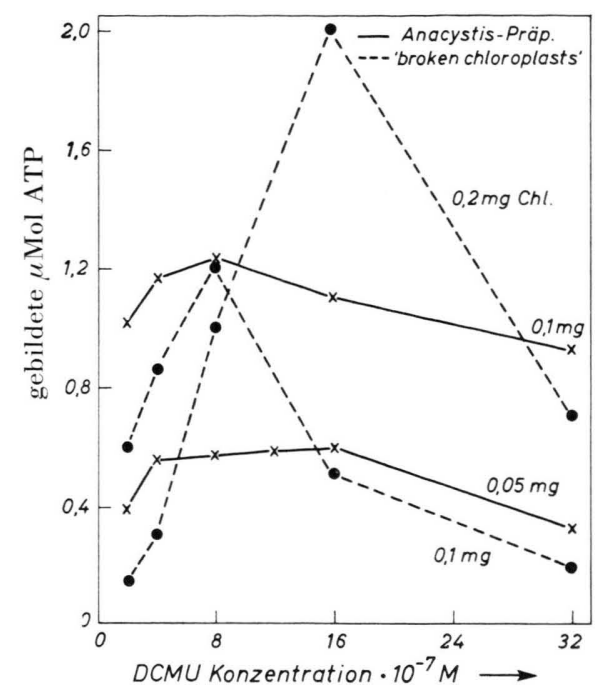

Abb. 1. DCMU-Abhängigkeit der Ferredoxin-katalysierten cyclischen Photophosphorylierung in Anacystis und in Chloroplasten. Reaktionsbedingungen: a) Algen: Präparation $\mathrm{N}_{\mathrm{Lys}}$, 1,5 ml Reaktionsvolumen, $35 \mathrm{~m} \mu \mathrm{Mol}$ Ferredoxin aus Spinat, pH 7,0, 30 Min. im Rotlicht (RGI-Filter) bei $25^{\circ} \mathrm{C}$. b) „,broken chloroplasts ": $\mathrm{P}_{1} \mathrm{~s}_{1}, 1,5 \mathrm{ml}$ Reaktionsvolumen, $130 \mathrm{~m} \mu \mathrm{Mol}$ Ferredoxin aus Spinat, pH 8,5, 15 Min. im Rotlicht (RGIFilter) bei $15^{\circ} \mathrm{C}$.

kaum notwendig erscheint. Wenn auch die Ferredoxin-katalysierte cyclische Photophosphorylierung in dem System aus Anacystis nur schwach DCMUabhängig ist, so zeigt die geringe Stimulierung der Reaktion durch DCMU doch, daß es sich bei ihr um eine cyclische und nicht um eine pseudocyclische Photophosphorylierung handelt, wie noch ausführlicher dargestellt wird.

Wie in isolierten Chloroplasten ${ }^{21}$ werden auch in dem System aus Anacystis zur optimalen Reaktion höhere Konzentrationen an Ferredoxin für die cyclische Photophosphorylierung als für die NADP. Reduktion benötigt (Abb. 2). Während lyophilisierte und Lysozym-behandelte Algen für die NADP. Reduktion sättigende Mengen an Ferredoxin enthalten und eine Abhängigkeit der NADP-Reduktion von Ferredoxin erst nach Extraktion der Algen deutlich wird ${ }^{10}$, läßt sich bereits in nicht extrahierten Algen eine Stimulierung der cyclischen ATPBildung durch Zusatz von Ferredoxin zeigen ${ }^{10}$. Wie aus Abb. 2 deutlich wird, ist in extrahierten Algen

20 D. I. Arnon, in: Currents of Photosynthesis, Hrsg. J. B. Thomas u. J. C. Goodheer, A. Donker-Publisher, Rotterdam, 1966, Diskussion, S. 479.

21 D. I. Arnon, International Congress of Photosynthesis Research, Freudenstadt, im Druck. 


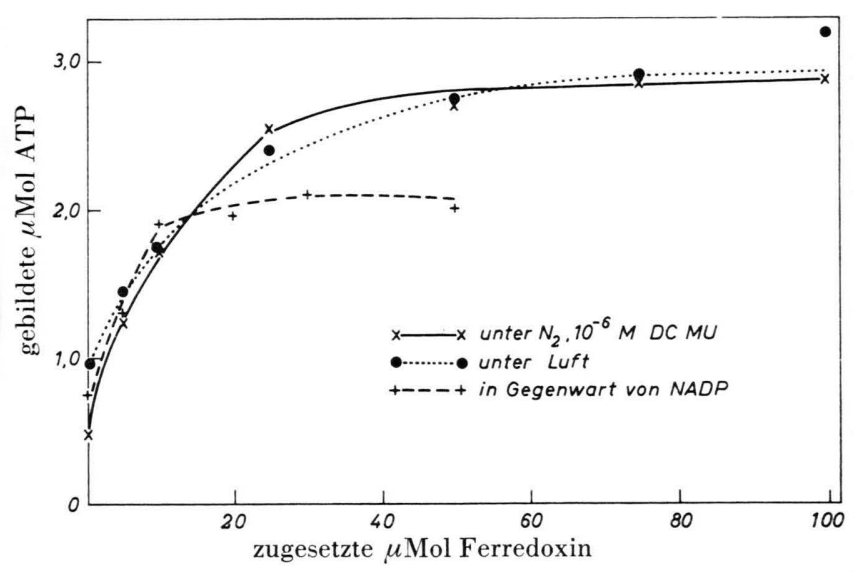

Abb. 2. Stimulierung der photosynthetischen ATP-Bildung in Anacystis durch Ferredoxin. Reaktionsbedingungen: $3 \mathrm{ml}$ Reaktionsvolumen, Algen $\left(\mathrm{R}_{2}\right)$ mit $0,2 \mathrm{mg}$ Chl., $\mathrm{pH}$ 7,0 mit Ausnahme des NADP-Systems ( $\mathrm{pH} 8,0), 20$ Min. Licht bei 35000 Lux und $25^{\circ} \mathrm{C}$.

die Sättigungskonzentration an Ferredoxin für die Ferredoxin-katalysierte cyclische Photophosphorylierung etwa 6-8-mal höher als für die NADP. Reduktion. Im Gegensatz zu den Verhältnissen in isolierten Chloroplasten ${ }^{2}$ sind die ermittelten ATP. Werte in der Ferredoxin-katalysierten cyclischen Photophosphorylierung der Präparation aus Ana-

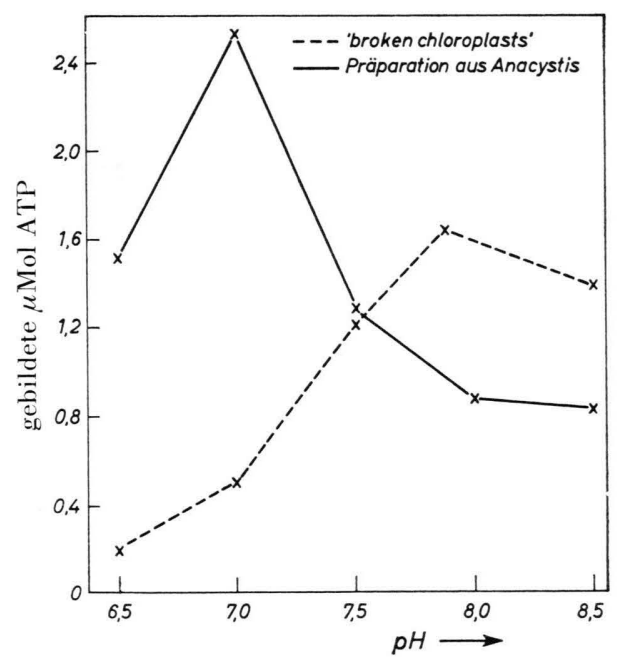

Abb. 3. pH-Abhängigkeit der Ferredoxin-katalytischen cyclischen Photophosphorylierung in Anacystis und in Chloroplasten. Reaktionsbedingungen: a) Algen: Präparation $\mathrm{N}_{\text {Lys }}$ mit 0,1 mg Chl., 1,5 ml Reaktionsvolumen, $35 \mathrm{~m} \mu$ Mol Ferredoxin aus Spinat, $8 \cdot 10^{-7} \mathrm{M}$ DCMU, 30 Min. im Rotlicht (RGIFilter) bei $25^{\circ} \mathrm{C}$. b) „broken chloroplasts“: $\mathrm{P}_{1} \mathrm{~s}_{1}$ mit $0,1 \mathrm{mg}$ Chl., 1,5 ml Reaktionsvolumen, $130 \mathrm{~m} \mu \mathrm{Mol}$ Ferredoxin aus Spinat, $4 \cdot 10^{-7} \mathrm{M}$ DCMU, 15 Min. im Rotlicht (RGI-Filter) bei $15^{\circ} \mathrm{C}$. cystis höher als in der offenkettigen Photophosphorylierung mit NADP als Elektronenakzeptor.

Unterschiedlich ist das $\mathrm{pH}$-Optimum in der Ferredoxin-katalysierten cyclischen Photophosphorylierung in Blaualgen und in Chloroplasten. KROGMANN stellte für die cyclischen Photophosphorylierungen mit PMS oder Menadion und FMN als Kofaktoren in einer Präparation aus Anabaena cylindrica ein pH-Optimum um 7,0 fest ${ }^{5}$. Aus Abb. 3 wird deutlich, daß auch die Ferredoxin-katalysierte cyclische Photophosphorylierung in lyophilisierter und Lysozym-behandelter Anacystis ein scharfes Optimum bei $\mathrm{pH} \mathrm{7,0} \mathrm{hat.} \mathrm{Im} \mathrm{Vergleich} \mathrm{dazu} \mathrm{hat} \mathrm{die} \mathrm{Ferredoxin-}$ katalysierte cyclische Photophosphorylierung isolierter Chloroplasten ihre maximale Rate bei einem $\mathrm{pH}$-Wert von etwa 8,0, jedoch ist das Maximum im pH-Bereich 7,5-8,5 nicht scharf ausgeprägt.

TAGAWA et al. ${ }^{3}$ berichteten, daß in isolierten Chloroplasten nur die Ferredoxin-katalysierte cyclische Photophosphorylierung, nicht aber die cyclischen Photophosphorylierungen mit anderen Kofaktoren oder die offenkettige Photophosphorylierung Antimycin-empfindlich ist. Auch in dem in Abb. 4 dargestellten Versuch wird die Reaktion

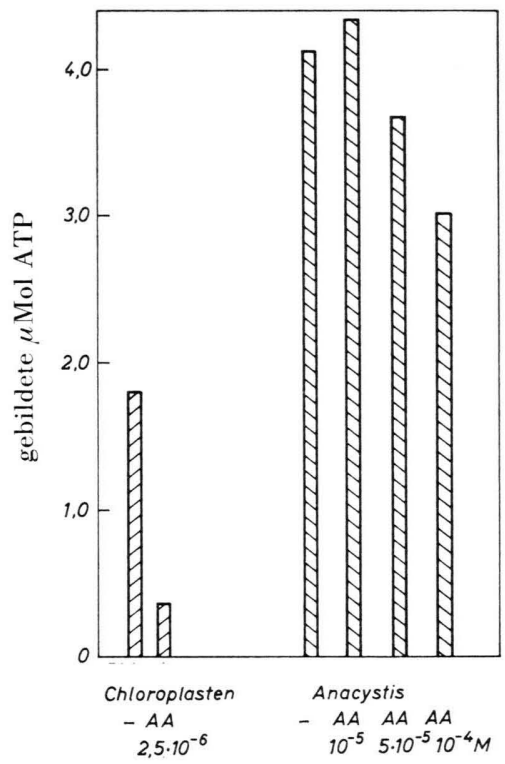

Abb. 4. Wirkung von Antimycin A auf die Ferredoxin-katalysierten cyclischen Photophosphorylierungen in Anacystis und in Chloroplasten. Reaktionsbedingung: a) Algen: Präparation $\mathrm{R}_{2}$ mit $0,2 \mathrm{mg}$ Chl., $3 \mathrm{ml}$ Reaktionsvolumen, 100 $\mathrm{m} \mu \mathrm{Mol}$ Ferredoxin Anacystis, $\mathrm{pH} 7,0,10^{-6} \mathrm{M}$ DCMU, 20 Min. Licht bei 35000 Lux und $25^{\circ} \mathrm{C}$. b) „broken chloroplasts": $\mathrm{P}_{1} \mathrm{~s}_{1}$ mit $0,2 \mathrm{mg}$ Chl., 1,5 ml Reaktionsvolumen, 100 m $u$ Mol Ferredoxin aus Anacystis, $8 \cdot 10^{-7}$ M DCMU, 15 Min. im Rotlicht (RGI-Filter) bei $15^{\circ} \mathrm{C}$. 
durch $2,5 \cdot 10^{-6} \mathrm{M}$ Antimycin A fast vollständig gehemmt. Überraschenderweise ist im Gegensatz dazu die Ferredoxin-katalysierte cyclische Photophosphorylierung in lyophilisierter Anacystis unempfindlich gegen diesen Hemmstoff (Abb. 4). Erst bei einer Konzentration von $10^{-4} \mathrm{M}$ Antimycin A läßt sich eine geringe Hemmung der Ferredoxin-katalysierten cyclischen Photophosphorylierung nachweisen, allerdings wird bei dieser hohen Konzentration auch die NADP-Reduktion im gleichen Maße gehemmt. Die Ferredoxin-katalysierte cyclische Photophosphorylierung in Anacystis wird auch dann nicht empfindlicher gegen Antimycin, wenn die Versuchsbedingungen variiert werden (vgl. dazu l.c. ${ }^{22,23}$ ). Insbesondere läßt sich bei geringen Lichtintensitäten, bei denen der Elektronentransport die Phosphorylierungsrate limitiert, in der Präparation aus Anacystis keine verstärkte Hemmung der Ferredoxin-katalysierten cyclischen Photophosphorylierung feststellen (Tab. 2).

Nach Trebst und Burba ${ }^{24}$ hemmt Disulfodisalicylidenpropandiamin (DSPD) nur die Ferredoxin-abhängigen photosynthetischen Reaktionen, insbesondere aber nicht die cyclischen Photophosphorylierungen mit anderen Kofaktoren als Ferredoxin. Die Wirkung von DSPD auf die Ferredoxinkatalysierten cyclischen Photophosphorylierungen wurde bislang nicht getestet und wird aus Tab. 3 deutlich. Sowohl in Chloroplasten als auch in dem

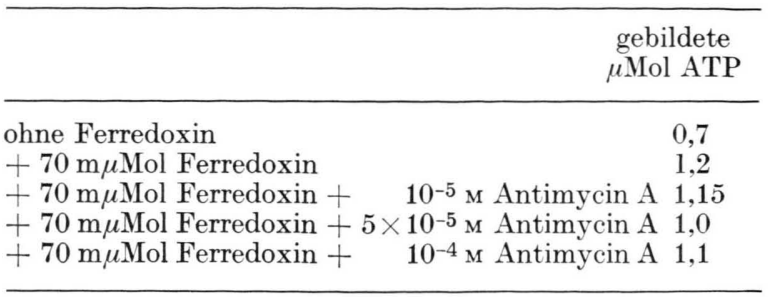

Tab. 2. Wirkung von Antimycin A auf die Ferredoxin-katalysierte cyclische Photophosphorylierung in Anacystis bei niedriger Lichtintensität. Reaktionsbedingungen: 3 ml Reaktionsvolumen, Algen $\left(R_{2}\right)$ mit $0,2 \mathrm{mg}$ Chl., Ferredoxin aus Anacystis, $\mathrm{pH} 7,0,10^{-6} \mathrm{M}$ DCMU, 30 Min. Licht bei 3000 Lux und $25^{\circ} \mathrm{C}$.

zellfreien System aus Anacystis hemmt DSPD sowohl die NADP-Reduktion als auch die Ferredoxinkatalysierte cyclische Photophosphorylierung. Dagegen werden die Ferredoxin-abhängigen pseudocyclischen Photophosphorylierungen (s.u.) durch den Hemmstoff nicht beeinflußt.

In isolierten Chloroplasten stimuliert Ferredoxin eine pseudocyclische Photophosphorylierung. Bei dieser Reaktion wird das photosynthetisch reduzierte Ferredoxin durch den Sauerstoff, der aus der Wasserspaltung entsteht, reoxidiert. Obwohl sich nur eine ATP-Bildung und kein Gaswechsel messen läßt, wird bei dieser Reaktion ATP natürlich nicht im cyclischen sondern im offenkettigen Elektronentransport gebildet $\left(\right.$ l.c. $\left.^{3,25}\right)$. Unterscheiden lassen sich cyclische und pseudocyclische Photophosphorylie-

\begin{tabular}{|c|c|c|c|c|c|}
\hline & \multicolumn{2}{|c|}{$\begin{array}{c}\text { unter } \mathrm{N}_{2} \text { unter Luft } \\
\text { gebildete }\end{array}$} & \multicolumn{3}{|c|}{ NADP-Reduktion } \\
\hline & $\mu \mathrm{Mol}$ & $\mu \mathrm{Mol}$ & $\mu$ Atome & $\mu \mathrm{Mol}$ & $\mu \mathrm{Mol}$ \\
\hline & ATP & ATP & $\mathrm{O}$ & $\mathrm{NADPH}_{2}$ & ATP \\
\hline \multicolumn{6}{|l|}{ a) Anacystis-Präparation } \\
\hline ohne Fd. & 0,85 & 1,6 & 1,0 & 1,2 & 0,7 \\
\hline+ Fd. & 4,1 & 4,1 & 3,0 & 3,3 & 2,15 \\
\hline$+\mathrm{Fd} .+5 \cdot 10^{-4} \mathrm{M}$ DSPD & 2,0 & 4,25 & 1,7 & 1,5 & 1,45 \\
\hline$+\mathrm{Fd} .+2 \cdot 10^{-3} \mathrm{M} \mathrm{DSPD}$ & 0,8 & 3,0 & 0,5 & 0,6 & 0,75 \\
\hline $\begin{array}{l}\text { b) Chloroplasten } \\
\text { ohne Fd. }\end{array}$ & \multicolumn{4}{|c|}{ b) Chloroplasten } & 0.4 \\
\hline$+\mathrm{Fd}$ & 0,9 & 2,2 & 2,3 & 2,1 & 2,05 \\
\hline$+\mathrm{Fd} .+5 \cdot 10^{-4} \mathrm{M} \mathrm{DSPD}$ & 0,15 & 1,8 & 1.0 & 0,8 & 1,4 \\
\hline$+\mathrm{Fd} .+2 \cdot 10^{-3} \mathrm{M} \mathrm{DSPD}$ & 0,05 & 1,9 & 0,3 & 0,1 & 0,45 \\
\hline
\end{tabular}

Tab. 3. Wirkung von Disalicylidenpropandiamin auf die Ferredoxin-stimulierten ATP-Bildungen in Stickstoff und in Luft und auf die NADP-Reduktion. Reaktionsbedingungen: a) Anacystis: Präparation $\mathrm{R}_{2}$ mit 0,2 mg Chl., 3 ml Reaktionsvolumen; $\mathrm{pH} 7,0,0,1 \mu \mathrm{Mol}$ Ferredoxin Anacystis, $10^{-6} \mathrm{M}$ DCMU im cyclischen System; pH 8,0,6 $\mu$ Mol NADP. $20 \mathrm{~m} \mu \mathrm{m}$ Ferredoxin im NADP-System; 20 Min. Licht bei 350000 Lux (ohne RGI-Filter) und $25^{\circ} \mathrm{C}$. b) Chloroplasten: $\mathrm{P}_{1} \mathrm{~s}_{1} \mathrm{mit} 0,1 \mathrm{mg}$ Chl., $1,5 \mathrm{~m}$ ' Reaktionsvolumen, $\mathrm{pH} 8,2 ; 0,1 \mu \mathrm{Mol}$ Ferredoxin Spinat, $4 \cdot 10^{-6} \mathrm{M}$ DCMU im cyclischen System; $6 \mu \mathrm{Mol}$ NADP, $20 \mathrm{~m} \mu \mathrm{Mol}$ Ferredoxin im NADP-System; 15 Min. Licht bei $15{ }^{\circ} \mathrm{C}$, RGI-Filter.

22 S. Izawa, T. N. Conolly, G. D. Winget u. N. E. Good, in: Brookhaven Symposia in Biology 19, 169 [1966].

23 .. C. Wessels, in der Diskussion zu 1. c. ${ }^{20}$, S. 479.
24 A. Trebst u. M. Burba, Z. Pflanzenphysiol. 57, 419 [1967].

25 D. I. Arnon, H. Y. Tsujimoto u. B. D. McSwain, Proc. nat. Acad. Sci. USA 51, 1274 [1964]. 


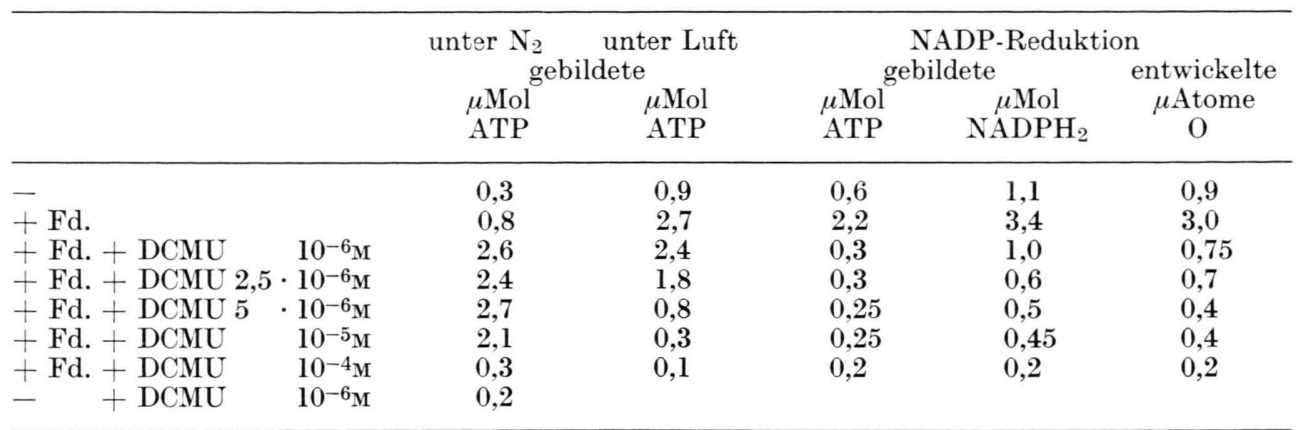

Tab. 4. Hemmung der Ferredoxin-abhängigen photosynthetischen Reaktionen in Anacystis durch DCMU. Reaktionsbedingungen: $3 \mathrm{ml}$ Reaktionsvolumen, Algen $\left(\mathrm{R}_{2}\right)$ mit 0,2 mg Chl.; $\mathrm{pH} 7,0,80 \mathrm{~m} \mu \mathrm{Mol}$ Ferredoxin Spinat im cyclischen System: $\mathrm{pH}$ 8,0, $6 \mu \mathrm{Mol}$ NADP, $20 \mathrm{~m} \mu \mathrm{Mol}$ Ferredoxin im NADP-System; 20 Min. Licht bei $35000 \mathrm{Lux}$ und $25^{\circ} \mathrm{C}(\mathrm{Fd}$. $=$ Ferredoxin).

rungen durch die Hemmbarkeit mit DCMU. Bei bestimmten DCMU-Konzentrationen und in Gegenwart von sättigenden Mengen an Ferredoxin wird im Spinatsystem die Ferredoxin-katalysierte pseudocyclische Photophosphorylierung fast vollständig gehemmt, die Ferredoxin-katalysierte cyclische Photophosphorylierung dagegen stimuliert (Tab. 4 in l.c. ${ }^{25}$ und Abb. 3 in l.c. ${ }^{3}$ ). Auch in lyophilisierter, Lysozym-behandelter und extrahierter Anacystis stimuliert Ferredoxin die photosynthetische ATP-Bildung unter aeroben Bedingungen. Die Rate dieser photosynthetischen ATP-Bildung ist genauso hoch wie die der Ferredoxin-katalysierten cyclischen Photophosphorylierung (vgl. Abb. 2). Aus Tab. 4 geht die Empfindlichkeit der Ferredoxin-stimulierten photosynthetischen ATP-Bildung in Luft, der NADPReduktion und der Ferredoxin-katalysierten cyclischen Photophosphorylierung gegen DCMU hervor. $2,5 \cdot 10^{-6} \mathrm{M}$ DCMU hemmt die NADP-Reduktion vollständig, die Ferredoxin-katalysierte cyclische Photophosphorylierung überhaupt nicht und die Ferredoxin-stimulierte ATP-Bildung in Luft nur zu etwa 25 Prozent. Die Ferredoxin-stimulierte ATPBildung in Luft ist im Anacystis-System also nicht ebenso empfindlich gegen DCMU wie im Chloroplastensystem, und das deutet bereits darauf hin, $\mathrm{da} \beta$ in Anacystis auch unter aeroben Bedingungen der größte Teil des ATP aus der cyclischen Photophosphorylierung stammt.

Alle getesteten ${ }^{26}$ pseudocyclischen Photophosphorylierungen in Chloroplasten erwiesen sich als Mehler-Reaktionen ${ }^{27}$. Bei einer Mehler-Reaktion wird ein photosynthetisch reduzierter Kofaktor unter $\mathrm{H}_{2} \mathrm{O}_{2}$-Bildung reoxidiert. Das gebildete $\mathrm{H}_{2} \mathrm{O}_{2}$

26 A. Trebst u. H. Eck, Z. Naturforschg. 16 b, 455 [1961]. kann im Chloroplastensystem durch Hemmung der endogenen Katalase mit $\mathrm{KCN}^{26}$ direkt gemessen werden. Auch bei der pseudocyclischen Photophosphorylierung mit Ferredoxin als Kofaktor wird in Chloroplasten $\mathrm{H}_{2} \mathrm{O}_{2}$ gebildet (Abb. $5 \mathrm{a}$ ). Nach $\mathrm{Zu}$ -

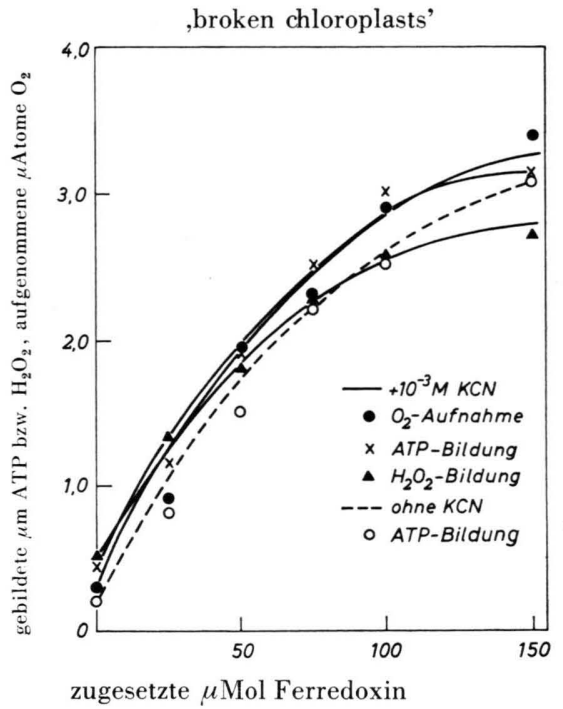

Abb. 5 a. Pseudocyclische Photophosphorylierung mit Ferredoxin als Kofaktor in Chloroplasten. Reaktionsbedingungen: $3 \mathrm{ml}$ Reaktionsvolumen, $\mathrm{P}_{1} \mathrm{~s}_{1}$ mit 0,2 mg Chl., $\mathrm{pH}$ 8,0, 15 Min. Licht bei 35000 Lux und $15^{\circ} \mathrm{C}$.

satz von $10^{-3} \mathrm{M} \mathrm{KCN}$ zum System werden genau so viele Äquivalente Sauerstoff aufgenommen wie ATP und wie $\mathrm{H}_{2} \mathrm{O}_{2}$ - in den Grenzen der $\mathrm{H}_{2} \mathrm{O}_{2}$-Bestimmungsmethode - gebildet werden. Im Gegensatz dazu läßt sich im Anacystis-System diese Stöchiometrie nicht feststellen. Denn in lyophilisierten, Lysozym-behandelten und extrahierten Algen sti-

27 A. H. Mehler, Arch. Biochem. Biophysics 33, 65 [1951]. 
muliert Ferredoxin in Gegenwart von Kaliumcyanid nur die ATP-Bildung nicht aber die Sauerstoffaufnahme und die $\mathrm{H}_{2} \mathrm{O}_{2}$-Bildung (Abb. $5 \mathrm{~b}$ ). Aus diesem Befund wird es deutlich, daß in Anacystis selbst unter aeroben Bedingungen ATP nach Zusatz von Ferredoxin über den cyclischen Elektronentransport gebildet wird.

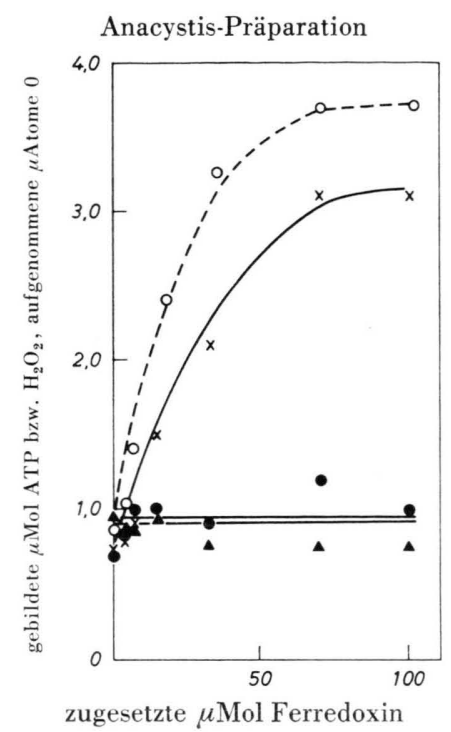

Abb. 5 b. Ferredoxin-stimulierte ATP-Bildung in Luft in Anacystis. Reaktionsbedingungen: $3 \mathrm{ml}$ Reaktionsvolumen, Algen $\left(\mathrm{R}_{2}\right)$ mit 0,2 mg Chl., pH 7,0, 20 Min. Licht bei 35000 Lux und $25^{\circ} \mathrm{C}$.

\section{Diskussion}

Bereits früher ${ }^{6,17}$ gelang in lyophilisierter und Lysozym-behandelter Anacystis der Nachweis aller wichtigen photosynthetischen Reaktionen, die in isolierten Chloroplasten bekannt geworden sind. So wurden in dem zellfreien System aus Anacystis eine photosynthetische Sauerstoffentwicklung im Ferricyanid- oder NADP-System mit gleichzeitiger ATP-Bildung und cyclische bzw. pseudocyclische Photophosphorylierungen mit verschiedenen artifiziellen Kofaktoren wie PMS oder Menadion beschrieben. In der Präparation läßt sich - wie hier berichtet wird - auch eine durch Ferredoxin katalysierte cyclische Photophosphorylierung nachweisen, die sich in ihren Eigenschaften erheblich von der Ferredoxin-katalysierten cyclischen Photophosphorylierung isolierter Chloroplasten unterscheidet. Wie bereits ausgeführt, läßt sich die Ferredoxin-katalysierte cyclische Photophosphorylierung isolierter Chloroplasten dann nachweisen, wenn sich die an ihr beteiligten Kofaktoren in einem bestimmten, genau einzustellenden Redoxzustand befinden ${ }^{18,19}$. Dieses „poising“ spielt im Anacystis-System eine geringere Rolle, wie an der geringen Empfindlichkeit der Reaktion gegen DCMU gesehen werden kann. Während in den Versuchen mit „broken chloroplasts " für die cyclische Photophosphorylierung es sich als günstig erwies, Rotlichtfilter zwischen der Lichtquelle und den Warburg-Gefäßen zu verwenden $^{2,10}$, hemmt Weißlicht (ohne Filter) die ATP-Bildung in der Ferredoxin-katalysierten cyclischen Photophosphorylierung nicht; wegen der höheren Lichtintensitäten werden im Weißlicht sogar höhere Raten als im Rotlicht in der Anacystis-Präparation gemessen (vgl. l.c. ${ }^{10}$ ). Die Ferredoxinkatalysierten cyclischen Photophosphorylierungen in Chloroplasten und Blaualgen unterscheiden sich weiterhin in ihrem unterschiedlichen $\mathrm{pH}$-Optimum und in ihrer Empfindlichkeit gegen Antimycin A. Vor allem aus der Antimycin-Hemmung der Ferredoxinkatalysierten cyclischen Photophosphorylierung werden wichtige Schlüsse gezogen. In isolierten Chloroplasten wird in einem bestimmten Konzentrationsbereich nur die Photophosphorylierung mit Ferredoxin nicht aber die mit anderen Kofaktoren von Antimycin gehemmt. In vivo sind von der Photophosphorylierung abhängige Reaktionen wie etwa die anaerobe, DCMU-unempfindliche und lichtabhängige Glucoseassimilation von Algen Antimycin-empfindlich ${ }^{28}$. Daher wird aus der Antimycin-Wirkung geschlossen, daß Ferredoxin der Katalysator der cyclischen Photophosphorylierung in vivo ist ${ }^{2}$. Da in der Elektronentransportkette der Mitochondrien Antimycin zwischen Cytochrom b und e hemmt, vermutet ARNON ${ }^{18}$ diese Kofaktoren (Cytochrom $b_{6}$ bzw. Cytochrom $f$ der Chloroplasten) in der Elektronentransportkette der Ferredoxin-katalysierten cyclischen Photophosphorylierung. Die Blaualge Anacystis ist ein Beispiel, bei dem die Ferredoxin-katalysierte cyclische Photophosphorylierung nicht von Antimycin A gehemmt wird, obwohl in Anacystis Cytochrom $b_{6}$ und Cytochrom $f$ nachgewiesen wur$\operatorname{den}^{29,7}$. Es ist daher unklar, welche Kofaktoren außer Ferredoxin an der cyclischen Photophosphorylierung sowohl der Blaualgen als auch der Chloroplasten beteiligt sind und was der Grund für die unterschiedliche Empfindlichkeit der Reaktionen gegen Antimycin ist. Nach Izawa et al. ${ }^{22}$ soll die Hemmstelle von Antimycin sich nicht spezifisch lokalisieren lassen, sondern Antimycin soll immer dann 
hemmen, wenn der Elektronentransport die Rate des Phosphorylierungssystems begrenzt. Wenn diese Behauptung richtig ist, könnte angenommen werden, daß im Gegensatz zum Chloroplastensystem in Anacystis der Elektronentransport in der Ferredoxin-katalysierten cyclischen Photophosphorylierung nicht limitierend ist. Darauf könnte die im Vergleich zur offenkettigen Photophosphorylierung höhere Rate in der Ferredoxin-katalysierten cyclischen Photophosphorylierung hindeuten. Allerdings stellt man bei stark limitierenden Lichtintensitäten und damit bei limitierendem Elektronenfluß keine Hemmung der Ferredoxin-katalysierten cyclischen Photophosphorylierung durch Antimycin fest, so daß die Unempfindlichkeit der Ferredoxin-katalysierten cyclischen Photophosphorylierung gegen Antimycin in Anacystis andere Ursachen haben muß.

Außer Antimycin A erwies sich unter den getesteten Hemmstoffen Disalicylidenpropandiamin als wichtig. Nach Trebst und Burba ${ }^{24}$ sind unter allen untersuchten nur die Ferredoxin-abhängigen Reaktionen wie die NADP- oder die Nitritreduktion empfindlich gegen DSPD; andere photosynthetische Reaktionen, insbesondere cyclische Photophosphorylierungen mit Menadion oder PMS als Kofaktoren werden nicht beeinflußt. Da DSPD in intakter Chlorella die photosynthetische $\mathrm{CO}_{2}$-Fixierung wesentlich weniger beeinflußt als die lichtabhängige, DCMU unempfindliche, anaerobe Glucoseassimilation, vermuten die Autoren ${ }^{24}$, daß DSPD ein spezifischer Hemmstoff der Ferredoxin-katalysierten cyclischen Photophosphorylierung ist. Wie aus den hier berichteten Ergebnissen hervorgeht, hemmt DSPD tatsächlich die Ferredoxin-katalysierten cyclischen Photophosphorylierungen sowohl in isolierten Chloroplasten als auch im Anacystis. System. Damit ist DSPD der zweite Hemmstoff neben Antimycin in höheren Pflanzen - aus dessen Wirkung eindeutig gefolgert werden kann, daß Ferredoxin der Kofaktor der cyclischen Photophosphorylierung in vivo ist. Allerdings hemmt DSPD überraschenderweise weder die Ferredoxin-katalysierte pseudocyclische Photophosphorylierung in Chloroplasten noch die Ferredoxin-stimulierte ATPBildung in Luft im Anacystis-System. Die Hemm-

28 O. Kandler u. W. Tanner, Ber. dtsch. bot. Ges. 79, 48 [1966].

29 R. W. Holton u. J. Myers, Science [Washington] 142, 234 [1963]. stelle des DSPD läßt sich damit nicht mehr eindeutig zwischen Pigmentsystem I und Ferredoxin (vgl. l.c. $\left.{ }^{24}\right)$ festlegen. Trebst und Burba ${ }^{24}$ nehmen an, daß DSPD photosynthetisch reduziert wird und dadurch hemmt; es könnte weiter vermutet werden, daß unter aeroben Bedingungen die Reduktion von DSPD und damit die Hemmwirkung unterbleibt. Jedenfalls ist der Mechanismus der Hemmwirkung von DSPD noch unklar und bedarf weiterer Untersuchungen.

In isolierten Chloroplasten ist die Sättigungskonzentration an Ferredoxin für die cyclische Photophosphorylierung etwa 10-mal höher als für die offenkettige Photophosphorylierung mit NADP als Elektronenakzeptor ${ }^{21}$. Es wird eingewendet, diese hohe Ferredoxin-Konzentration könne kaum physiologisch und damit Ferredoxin nicht der Kofaktor der cyclischen Photophosphorylierung in vivo sein ${ }^{\mathbf{3 0}}$. Lyophilisierte und Lysozym-behandelte Zellen von Anacystis enthalten sättigende Mengen an Ferredoxin für die NADP-Reduktion, nicht aber für die cyclische ATP-Bildung ${ }^{\mathbf{1 0}}$. Nach der anschließenden Extraktion der Algen läßt sich auch eine Abhängigkeit der NADP-Reduktion von Ferredoxin zeigen, und zur Sättigung der cyclischen Photophosphorylierung werden etwa 6-8-mal mehr Ferredoxin als für die NADP-Reduktion benötigt. Obwohl also auch im Anacystis-System die Sättigungskonzentration an Ferredoxin für die cyclische Photophosphorylierung höher als für die NADP-Reduktion ist, sehen wir darin keinen Einwand gegen Ferredoxin als Kofaktor der cyclischen Photophosphorylierung in vivo. Denn einerseits ist nicht bekannt, mit welcher Rate überhaupt die cyclische Photophosphorylierung in vivo ablaufen muß, andererseits können über Gehalt an Ferredoxin in intakten Chloroplasten oder Algen keine Aussagen gemacht werden, da durch die Isolierung notwendigerweise die photosynthetischen Strukturen und Kofaktoren verdünnt werden. Außerdem sind die Sättigungskonzentrationen für die Kofaktoren der cyclischen Photophosphorylierungen mit PMS, DCPIP, FMN oder Menadion eher noch höher als für Ferredoxin (vgl. l.c. ${ }^{30}$ ). Neben Ferredoxin käme noch Phytoflavin als Kofaktor der cyclischen Photophosphorylierung in

30 M. Avron u. J. Neumann, in: Annual Rev. Plant Physiol. 19, 137 [1968]. 
Blaualgen in Frage, da Phytoflavin Ferredoxin in allen getesteten Reaktionen vollständig ersetzen kann, wie wir berichteten ${ }^{31,32,10}$. Allerdings wird Phytoflavin nur unter Eisenmangelbedingungen gebildet ${ }^{31,32}$, so daß unter Normalbedingungen nur Ferredoxin Kofaktor der cyclischen Photophosphorylierung sein kann. In den vitro-Versuchen ersetzen sich Ferredoxin aus Spinat und aus Anacystis vollständig ${ }^{31,33,10}$, obwohl im Ferredoxin aus Anacystis doppelt so viel Eisen wie labiler Schwefel enthalten sein soll ${ }^{34}$.

Die Raten der Ferredoxin-katalysierten cyclischen Photophosphorylierung in Chloroplasten und in Anacystis können kaum miteinander verglichen werden, da sich in unseren Versuchen die Ferredoxinkatalysierte cyclische Photophosphorylierung in Chloroplasten nur bei limitierendem Licht messen ließ (vgl. l. c. ${ }^{10}$ ). Die Ferredoxin-katalysierte cyclische Photophosphorylierung in Anacystis wurde bei hohen, nicht limitierendem Licht gemessen, und die Rate ist in dem Reaktionsansatz mit dem vergleichsweise großen Reaktionsvolumen von $3 \mathrm{ml}$ mit bis zu $80 \mu \mathrm{Mol} \mathrm{ATP} / \mathrm{h} / \mathrm{mg}$ Chlorophyll beträchtlich. Im offenkettigen Elektronentransport mit NADP als Akzeptor ist in lyophilisierter und Lyso-

31 A. Trebst u. H. Bothe, Ber. dtsch. bot. Ges. 79, 44 [1966].

32 H. Bothe, International Congress of Photosynthesis Research, Freudenstadt 1968, im Druck. zym-behandelter Anacystis die ATP-Bildung geringer, dort ist das Verhältnis von $\mathrm{NADPH}_{2}$ - und ATPBildung nur $2: 1$, und es läßt sich keine Kopplung von ATP-Bildung und Elektronentransport zeigen ${ }^{10}$ (vgl. auch l.c. $\left.{ }^{8}\right)$. Es ist nicht auszuschließen, daß bei der Herstellung der Präparation aus Anacystis die Kopplung von ATP-Bildung und Elektronentransport verloren geht. Aus den geschilderten Versuchsbedingungen kann aber vermutet werden, daß das photosynthetisch zu bildende ATP in Anacystis im Vergleich zu dem der Chloroplasten höherer Pflanzen wesentlich mehr aus dem cyclischen als aus dem offenkettigen Elektronentransport angeliefert wird. Die gut entwickelte und stabile cyclische Photophosphorylierung in Blaualgen erinnert an die Verhältnisse in phototrophen Bakterien, dagegen sind die Elektronencarrier der photosynthetischen Reaktionen in Blaualgen weitgehend ähnlich wie in höheren Pflanzen, wie aus den Arbeiten mit BlaualgenPräparationen übereinstimmend hervorgeht.

Herrn Professor Dr. A. Trebst danke ich für viele helfende Diskussionen, und der Deutschen Forschungsgemeinschaft bin ich für die Unterstützung der Arbeit durch Sachbeihilfen dankbar.

33 M. C. W. Evans, D. O. Hall, H. Bothe u. F. R. Whatley, Biochem. J. 110, 485 [1968].

34 T. Yamanaka, S. Takaenami, K. Wada u. K. OKunuki, Biochim. biophysica Acta [Amsterdam] 180, 196 [1969]. 